\title{
Mammalian Two-Hybrid System: A Complementary Approach to the Yeast Two-Hybrid System
}

\author{
Ying Luo, Anna Batalao, Helen Zhou and Li Zhu \\ CLONTECH Laboratories, Palo Alto, CA, USA
}

BioTechniques 22:350-352 (February 1997)

\begin{abstract}
Here we demonstrate the use of a mammalian two-hybrid system to study protein-protein interactions. Like the yeast two-hybrid system, this is a genetic, in vivo assay based on the reconstitution of the function of a transcriptional activator. In this system, one protein of interest is expressed as a fusion to the Gal4 DNA-binding domain and another protein is expressed as a fusion to the activation domain of the VP16 protein of the herpes simplex virus. The vectors that express these fusion proteins are cotransfected with a reporter chloramphenicol acetyltransferase (CAT) vector into a mammalian cell line. The reporter plasmid contains a cat gene under the control of five consensus Gal4 binding sites. If the two fusion proteins interact, there will be a significant increase in expression of the cat reporter gene. Previously, it was reported that mouse p53 antitumor protein and simian virus 40 large $T$ antigen interact in a yeast two-hybrid system. Using a mammalian two-hybrid system, we were able to independently confirm this interaction. The mammalian two-hybrid system can be used as a complementary approach to verify proteinprotein interactions detected by a yeast two-hybrid system screening. In addition, the mammalian two-hybrid system has two main advantages: (i) Assay results can be obtained within 48 h of transfection, and (ii) protein interactions in mammalian cells may better mimic actual in vivo interactions.
\end{abstract}

\section{INTRODUCTION}

The yeast two-hybrid system has been one of the most successfully used approaches to identify and characterize protein-protein interactions (7). In this system, the Gal4 transcription factors can be functionally and physically divided into a DNA-binding domain and a transcription-activation domain. Linking these two domains to other proteins results in chimeric proteins with either specific DNA-binding ability (DNA-binding domain fusion protein) or a nonspecific transcription-activation function (activation-domain fusion protein). Interactions between these two chimeric proteins in yeast can bring the Gal4 activation domain and the DNAbinding domain together and reconstitute the Gal4 transcription-activation function. Any reporter gene under the transcriptional control of Gal4 binding sites can be activated by this specific protein-protein interaction.

Here, a mammalian two-hybrid system was developed using a strategy similar to that used in the yeast two-hybrid system $(2,3,5,6,8,11,12)$. Two chimeric proteins were expressed in mammalian cells from the simian virus 40 (SV40) promoter. Just as in the yeast two-hybrid system, the first bait hybrid was constructed by fusing the Gal4 DNA-binding domain to mouse p53. Instead of the Gal4 activation domain, the VP16 activation domain was fused to the SV40 T antigen to construct the second hybrid protein. In mammalian cells, the reporter gene was chloramphenicol acetyltransferase (cat) under the control of five copies of the Gal4 binding sites 
upstream of the E1b minimal promoter. Binding of p53 to SV40 T antigen brought the two hybrids together to activate cat gene expression.

Protein interactions in mammalian cells might mimic the in vivo situation better than in yeast cells. This may be due to protein folding, post-translational modification and cellular localization. After yeast two-hybrid system screening, other methods, such as in vitro binding assays, co-immunoprecipitation assays and function analysis of the cloned cDNA, are routinely used to rule out false-positive clones and verify protein-protein interactions (4). The mammalian two-hybrid system is another method that can be used to verify true-positive interaction and rule out false-positive clones. Compared to other available approaches, the mammalian two-hybrid system allows for easier and faster confirmation.

\section{MATERIALS AND METHODS}

\section{Plasmid Construction}

$\mathrm{pM}$, an SV40 promoter driving the Gal4 DNA-binding domain expression plasmid, was obtained from the laboratory of Sadowski (10). A multiple cloning site downstream from the Gal4 binding domain could be used to insert bait protein (Figure 1). pVP16 was constructed by adding the SV40 nuclear localization signal to the $\mathrm{N}$ terminus of the VP16 protein.

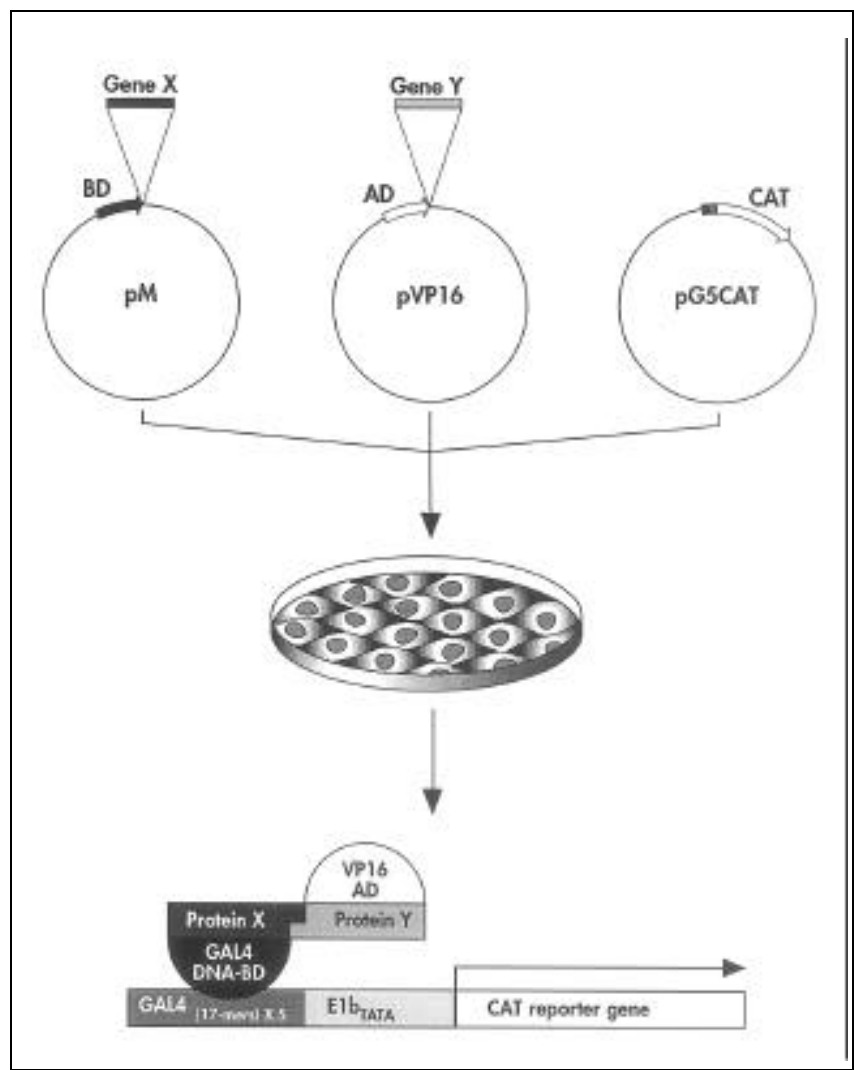

Figure 1. Flow chart for using the mammalian two-hybrid assay kit. pM and pVP16 were the two vectors used to construct hybrid proteins for expression in mammalian cells. pG5CAT is the CAT reporter plasmid under the control of five copies of the Gal4 binding sites. All three plasmids were cotransfected into mammalian cells, and then CAT assays were performed $48 \mathrm{~h}$ post-transfection. An interaction between protein $\mathrm{X}$ and protein $\mathrm{Y}$ results in a significantly elevated level of cat gene expression.
This was done by polymerase chain reaction (PCR) amplification of the VP16 activation domain by using a pair of oligonucleotides containing an SV40 nuclear localization signal at the $\mathrm{N}$ terminus. The PCR fragment was then inserted into the NheI/EcoRI site of $\mathrm{pM}$ to replace the Gal4 binding domain. A multiple cloning site, identical to that in $\mathrm{pM}$, was located downstream of the VP16 open reading frame for insertion of the partner protein. pM-53 was constructed by cutting out the p53 fragment of the pGAD53m and then inserting it in-frame into the EcoRI/SalI site of pM. pVP16-T was constructed by PCR amplification of the SV40 T antigen. The PCR fragment was then inserted into the pVP16 plasmid EcoRI/SalI site in-frame. pVP16CP was a negative control plasmid constructed by inserting a PCR-amplified polyoma virus VP3 fragment in-frame into the pVP16 EcoRI/SalI site. pM-VP16 was a positive control plasmid expressing a Gal4(binding domain)-VP16(activation domain) hybrid protein. pG5CAT contained a CAT reporter under the control of five copies of the Gal4 binding sites upstream of an E1b minimal promoter. Both pM-VP16 and pG5CAT were obtained from the laboratory of Sodowski (10).

\section{Cell Culture and Transfection}

HeLa cells were cultured in 10-cm dishes to $30 \%$ confluency in Dulbecco's modified Eagle medium (DMEM) plus $10 \%$ fetal calf serum. The $\mathrm{Ca}^{2+}$ transfection method was used in all of our experiments (1). After transfection, the cells were incubated for 12-24 $\mathrm{h}$ before adding fresh medium and were harvested within $48 \mathrm{~h}$ after transfection.

\section{CAT Assays}

We used a CAT ELISA kit (Boehringer Mannheim, Indianapolis, IN, USA) for all of our CAT assays. The assays were repeated at least twice to obtain an average number.

\section{RESULTS AND DISCUSSION}

It has been reported that SV40 $\mathrm{T}$ antigen is able to bind to p53 protein in the yeast two-hybrid system assay. To verify that SV40 T antigen was also able to bind to p53 in our mammalian two-hybrid system, we constructed two hybrid protein expression plasmids, $\mathrm{pM}-53$ and $\mathrm{pVP} 16-\mathrm{T}$. Whereas in the yeast two-hybrid system, the HIS3 and LacZ reporter genes were used, in this study, a cat reporter gene under the control of five copies of the Gal4 binding sites was used.

Unlike the yeast two-hybrid system, the mammalian twohybrid system is a transient assay. Three plasmids containing the cat reporter gene and two hybrid proteins (pG5CAT, pM53 and pVP16-T) had to be cotransfected into mammalian cells for the CAT assay. Since the promoter driving our hybrid protein expression was an SV40 early promoter, we used HeLa cells in our experiments; whereas, other laboratories used CV1 cells, Chinese hamster ovary (CHO) cells and Jurkat cells in similar systems $(2,3,5,6,8,11,12)$.

For better co-transfection efficiency, we chose the BES$\mathrm{Ca}^{2+}$ transfection method (1), which was a very efficient method for HeLa cell transfection. Although we also tried the DEAE-dextran method, a lower level of CAT activation was observed in those experiments. Forty to forty-eight hours after transfection, the cells were harvested for CAT assay. As 
shown in Figure 2, pM-53 and pVP16-T co-transfection generated about 58 -fold activation of CAT expression, compared with 1.5-fold by $\mathrm{pM}-53$ and $\mathrm{pVP16}-\mathrm{CP}$ co-transfection. This assay verified the binding of SV40 $\mathrm{T}$ antigen to mouse p53 protein in mammalian cells.

Although we were not aware of examples of protein-protein interactions identified in the yeast two-hybrid system that could not be repeated in the mammalian two-hybrid system, others observed that certain protein-protein binding in vitro was different from that in yeast (5). Therefore, we reasoned that since the post-translational modifications in yeast were different from those in mammalian cells, it was also quite plausible that the false-positive results generated by the yeast two-hybrid system cDNA library screening might be due to improper mammalian protein folding, phosphorylation, acylation and glycosylation in yeast. False-positive results in twohybrid screening could also be caused by binding of the two hybrid proteins to a common third endogenous yeast protein, instead of binding directly to each other. However, this third endogenous protein might not be present in mammalian cells at all. Therefore, using both the mammalian two-hybrid system and the yeast two-hybrid system might help to quickly eliminate false-positive clones.

Another advantage to using the mammalian two-hybrid system is that this assay is less time-consuming than the yeast two-hybrid system. Instead of waiting 3-4 days for yeast colonies to grow to a reasonable size for blue-color assay, the CAT assay could be done within $48 \mathrm{~h}$ of transfection. Quantitative results are also immediately available. In the yeast twohybrid system, the colony must be cultured in liquid medium for quantitative $\beta$-galactosidase analysis.

The yeast two-hybrid system is effective for cDNA library screening. The mammalian two-hybrid system, using the hygromycin drug resistance gene or the CD4 cell-surface marker as a reporter, may be explored further for this purpose (6).

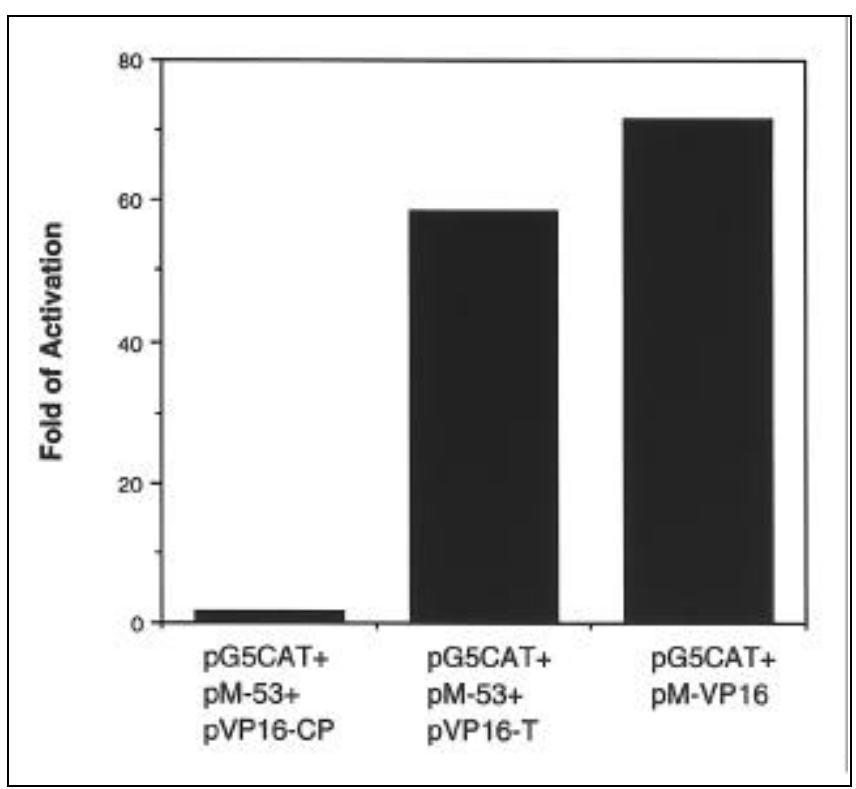

Figure 2. Demonstration of the interaction between p53 and the SV40 T antigen in HeLa cells by using the mammalian two-hybrid system. HeLa cells grown to $30 \%$ confluency were transfected by the calcium phosphate method with the indicated plasmids. Each transfection used $22 \mu \mathrm{g}$ of DNA (2 $\mu \mathrm{g}$ of pG5CAT and 10 or $20 \mu \mathrm{g}$ of each of the other plasmids). CAT assays were done using a CAT ELISA kit. The numbers shown are the fold of activation of cat gene expression.
However, other mammalian systems using luciferase or $\beta$ galactosidase cannot be used in cDNA library screening. At present, there is no very efficient mammalian cloning system available. It is possible that the mammalian one-hybrid system can be built in a way similar to the yeast one-hybrid system for the study of DNA-binding proteins (9).

\section{REFERENCES}

1.Ausubel, F.M., R. Brent, R.E. Kingston, D.D. Moore, J.G. Seidman, J.A. Smith and K. Struhl. 1995. Current Protocols in Molecular Biology. John Wiley \& Sons, New York.

2.Bogerd, H.P., R.A. Fridell, S. Madore and B.R. Cullen. 1995. Identification of a novel cellular cofactor for the Rev/Rex class of retroviral regulatory proteins. Cell 82:485-494.

3.Dang, C.V., J. Barrett, M. Villa-Garcia, L.M.S. Resar, G.J. Kato and E.R. Fearon. 1991. Intracellular leucine zipper interactions suggest cMyc heteraoligomerization. Mol. Cell. Biol. 11:954-962.

4.Durfee, T., K. Becherer, P.L. Chen, S.H. Yeh, Y. Yang, A.E. Kilburn, W.H. Lee and S.J. Elledge. 1993. The retinoblastoma protein associates with the protein phosphatase type 1 catalytic subunit. Genes Dev. 7:555569.

5.Fagan, R., K.J. Flint and N. Jones. 1994. Phosphorylation of E2F-1 modulates its interaction with the retinoblastoma gene product and the adenoviral E4 $19 \mathrm{kDa}$ protein. Cell 78:799-811.

6.Fearon, E.R., T. Finkel, M.L. Gillison, S.P. Kennedy, J.F. Casella, G.F. Tomaselli, J.S. Morrow and C.V. Dang. 1992. Karyoplasmic interaction selection strategy: a general strategy to detect protein-protein interaction in mammalian cells. Proc. Natl. Acad. Sci. USA 89:7958-7962.

7.Fields, S. and O. Song. 1989. A novel genetic system to detect proteinprotein interactions. Nature 340:245-247.

8.Hsu, H.L., I. Wadman and R. Baer. 1994. Formation of in vivo complexes between the TAL1 and E2A polypeptides of leukemic T cells. Proc. Natl. Acad. Sci. USA 91:3181-3185.

9.Luo, Y., S. Vijaychander, J. Stile and L. Zhu. 1996. Cloning and analysis of DNA-binding proteins by yeast one-hybrid and one-two-hybrid system. BioTechniques 20:564-568.

10.Sadowski, I., B. Bell and M. Hollis. 1992. GAL4 fusion vectors for expression in yeast or mammalian cells. Gene 118:137-141.

11.Takacs, A.M., T. Das and A.K. Banerjee. 1993. Mapping of interacting domain between the nucleocapsid protein and the phophorprotein of vescular stomatitis virus by using a two-hybrid system. Proc. Natl. Acad. Sci. USA 90:10375-10379.

12.Vasavada, H.A., S. Ganguly, F.J. Germino, Z.X. Wang and S.M. Weissman. 1991. A contingent replication assay for the detection of protein-protein interactions in animal cells. Proc. Natl. Acad. Sci. USA 88:10686-10690.

Address correspondence to Ying Luo, CLONTECH Laboratories, Inc., 1020 East Meadow Circle, Palo Alto, CA 94303-4230,USA. Internet: yluo@clontech.com 